\title{
The Kohlrausch function: properties and applications
}

\author{
R. S. Anderssen* Saiful A. Husain ${ }^{\dagger} \quad$ R. J. Loy ${ }^{\ddagger}$
}

(Received 8 August 2003; revised 8 May 2004)

\begin{abstract}
In a wide variety of applications, including the modelling of the glassy state of dense matter, non-exponential correlation functions in nuclear magnetic resonance, polymer dynamics, and bone and muscle rheology, Kohlrausch functions have proved to be more appropriate in modelling the associated relaxation and decay processes than the standard exponential function. However, mathematical results about this function, important for both computational and modelling endeavours, are spread over publications in several quite different areas of mathematics and science. The purpose of this paper is to review
\end{abstract}

\footnotetext{
${ }^{*}$ CSIRO, Mathematical and Information Sciences, GPO Box 664, Canberra, ACT 2601, Australia. mailto:Bob. Anderssen@csiro.au

${ }^{\dagger}$ CMA, Mathematical Sciences Institute, Australian National University, Canberra, ACT 0200, AustraliA. mailto:Pol.Haji@maths.anu.edu.au

${ }_{\ddagger}^{\ddagger}$ Mathematics Department, Mathematical Sciences Institute, Australian National University, Canberra, ACT 0200, AustraliA. mailto:Rick.Loy@maths.anu. edu. au

See http://anziamj.austms.org.au/V45/CTAC2003/Ande/home.html for this article, (c) Austral. Mathematical Soc. 2004. Published August 8, 2004. ISSN 1446-8735
} 
the key properties of Kohlrausch functions in a unified manner, which motivates its use in the modelling of molecular processes. Some representative applications and related computational issues are discussed.

\section{Contents}

1 Introduction

C801

2 Key properties of Kohlrausch functions

C803

2.1 Complete monotonicity and infinite divisibility . . . . . . C C803

2.2 Kohlrausch functions and stable distributions . . . . . . C C803

2.3 A Paley-Wiener property for support bounded on the left C805

3 Applications

C806

3.1 The oscillatory shear connection . . . . . . . . . . . C806

3.2 Large-scale polymer chain dynamics . . . . . . . . . . . C C811

3.3 Recovery of Kohlrausch parameters . . . . . . . . . . . C C812

3.4 Public domain algorithms . . . . . . . . . . . . . C813

References

C813

\section{Introduction}

Various authors [5, 16, 18, 21, 22, 24] observed that Kohlrausch (stretched exponential) functions, which, for fixed $\beta, 0<\beta<1$, and $\tau>0$, take the form

$$
K_{\tau, \beta}(t)=\exp \left(-(t / \tau)^{\beta}\right), \quad 0 \leq t<\infty,
$$

are often more appropriate in modelling relaxation processes in bone, muscles, dielectric materials, polymers and glasses than standard exponentials. In part, this is a consequence of the fact that, because a relaxation depends 
on the entire spectrum of relaxation times, its structure will be non-linear and not purely exponential. For example, Avellaneda et al. [3] showed that the probability distribution solutions of Burgers' equation, with a random stationary Gaussian as the initial condition, have tails that take the form of Kohlrausch functions.

As a result, Kohlrausch functions are now assumed by many to represent a "universal model" $[15,16]$. Functions of the form (1) were first proposed by R. Kohlrausch in 1854 in the study of creep in electric displacements $[4,12]$. Different names are used for Kohlrausch functions such as the Williams-Watts empiric dielectric relaxation functions, the $\mathrm{KWW}$ (Kohlrausch-Williams-Watts) functions, and the stretched exponentials.

From a computational techniques and applications perspective, there is currently no publication that considers the properties of Kohlrausch functions in a unified manner. Even some of the statements in the literature about its properties are imprecise, which the current article aims to correct. This paper therefore represents a first attempt to list the properties in a unified manner as well as motivate the use of Kohlrausch functions in the modelling of molecular processes in terms of some representative examples.

Of course, many of the properties of Kohlrausch functions hold for the standard exponential function. However, in this paper, the goal is to highlight theoretical and practical situations where Kohlrausch functions might be the more appropriate choice.

\section{Key properties of Kohlrausch functions}

\subsection{Complete monotonicity and infinite divisibility}

Kohlrausch functions $K_{\tau, \beta}(t)$ are a special case of a function of the form $\exp (-\theta(t)), 0 \leq t<\infty$, where 
1. $\theta(0)=0$, and

2. the derivative $\dot{\theta}(t)$ of $\theta(t)$ is completely monotone; that is, it satisfies

$$
(-1)^{n} \frac{d^{n} \dot{\theta}(t)}{d t^{n}} \geq 0, \quad \text { for all non-negative integers } n .
$$

The conditions 1 and 2 not only guarantee that $\exp (-\theta(t)$ ) (and hence Kohlrausch functions) are completely monotone, they are also the necessary and sufficient conditions for $\exp (-\theta(t))$ to be the Laplace transform of an infinitely divisible measure $d \mu(p)[1,2,7,8]$; namely,

$$
\exp (-\theta(t))=\int_{0}^{\infty} \exp (-t p) d \mu(p) .
$$

\subsection{Kohlrausch functions and stable distributions}

When $\theta(t)=t^{\beta}, 0<\beta \leq 1$, Pollard [20] established that equation (3) can be rewritten as

$$
\exp \left(-t^{\beta}\right)=\int_{0}^{\infty} \varphi(\beta, p) \exp (-t p) d p
$$

It has been known since the work of Lévy that $\varphi(\beta, p)$ is a stable distribution [7, e.g.]. Such distributions are known to be unimodal [25]. Various explicit formulas for $\varphi(\beta, p)$ have been determined. Doetsch [6] established

$$
\varphi\left(\frac{1}{2}, p\right)=\frac{1}{2 \pi^{1 / 2} p^{3 / 2}} \exp \left(-\frac{1}{4 p}\right) .
$$

Montroll and Bendler [16] established that

$$
\varphi\left(\frac{1}{3}, p\right)=\frac{x}{\pi} \sin \left(\frac{\pi}{3}\right) K_{1 / 3}(x),
$$


where

$$
x=2\left(\frac{1}{3 p^{1 / 3}}\right)^{3 / 2}
$$

and $K_{v}$ is the modified Bessel function of order $v$. Humbert [11] showed formally that

$$
\varphi(\beta, p)=-\frac{1}{\pi} \sum_{k=0}^{\infty} \frac{(-1)^{k}}{k !} \sin (\pi \beta k) \frac{\Gamma(\beta k+1)}{p^{\beta k+1}}, \quad 0<\beta \leq 1 .
$$

In particular, when $\beta=2 / 3$,

$$
\varphi\left(\frac{2}{3}, p\right)=-\frac{1}{2(3 \pi)^{1 / 2}} p^{-1} \exp \left(-\frac{2}{27 p^{2}}\right) W_{-1 / 2,-1 / 6}\left(-\frac{4}{27 p^{2}}\right),
$$

where $W_{u, v}(z)$ denotes the Whittaker function. Pollard [20] gave a rigorous proof for equation (8) by first noting that

$$
\varphi(\beta, p)=\frac{1}{2 \pi i} \int_{\gamma} \exp (z p) \exp \left(-z^{\beta}\right) d z
$$

for suitable $\gamma$, and then, using contour integration methods, established that $\gamma$ can be replaced by a contour $\sigma$ along the negative real axis traversed $-\infty \rightarrow 0 \rightarrow-\infty$ on either side of the cut, and hence

$$
\varphi(\beta, p)=\frac{1}{\pi} \int_{0}^{\infty} \exp (-p u) \exp \left(-u^{\beta} \cos (\pi \beta)\right) \sin \left(u^{\beta} \sin (\pi \beta)\right) d u .
$$

An alternative contour integration argument, using the imaginary axis, yields the following alternative form for Pollard's solution

$$
\varphi(\beta, p)=\frac{1}{\pi} \int_{0}^{\infty} \exp \left(-\omega^{\beta} \cos (\pi \beta / 2)\right) \cos \left(\omega p+\omega^{\beta} \sin \pi \beta / 2\right) d \omega .
$$

This appears to be a new representation for $\varphi(\beta, p)$. Various authors proposed computational procedures for the evaluation of the (Lévy) stable distribution function $\varphi(\beta, p)$, such as its unimodal structure $[4,9]$. In their work, Lindsey and Patterson [13] found the summation (8) easier to evaluate than the integral (11). 


\subsection{A Paley-Wiener property for support bounded on the left}

Paley and Wiener [19] established the following result: Given $\phi(t) \in L_{2}(R)$ with support bounded on the left, then

$$
\int_{-\infty}^{\infty} \frac{|\log | \widehat{\phi}(\omega)||}{1+\omega^{2}} d \omega<\infty
$$

where

$$
\widehat{\phi}(\omega)=\int_{-\infty}^{\infty} \phi(t) \exp (-i \omega t) d t
$$

is the Fourier transform of $\phi(t)$; and, conversely, if (13) holds for some $\widehat{\phi}(\omega) \in$ $L_{2}(R)$, then there is an $F(t) \in L_{2}(R)$, with support bounded on the left, such that $|\widehat{F}(\omega)|=|\widehat{\phi}(\omega)|$.

Because a Kohlrausch function is an $L_{2}(R)$ function with support bounded on the left, it follows that the Fourier transform of such a function satisfies (13). On the other hand, Ngai et al. [17] noted that

$$
\widehat{\Psi}_{\beta}(\omega)= \begin{cases}\exp \left(-\omega^{\beta}\right), & \omega \geq 0, \\ 0, & \omega<0,\end{cases}
$$

satisfies equation (13) when $0<\beta<1$, but not for the standard exponential situation when $\beta=1$. Thus, for $0<\beta<1$, there is an $F_{\beta} \in L_{2}(R)$, with support bounded on the left, such that $\left|\widehat{F}_{\beta}\right|=\left|\widehat{\Psi}_{\beta}\right|=\widehat{\Psi}_{\beta}$. Interestingly, $\widehat{\Psi}_{\beta}(\omega)$ and its inverse Fourier transform are both $L_{2}(R)$ functions with support bounded on the left. The applicability of the above Paley-Wiener result to the response of disperse mechanical and electrical systems has been debated in the literature [14]. 


\section{Applications}

\subsection{The oscillatory shear connection}

In the analysis of the oscillatory shear behaviour of a linear viscoelastic material, the storage and loss moduli are defined by

$$
G^{\prime}(\omega)=\omega \int_{0}^{\infty} G(\tau) \sin (\omega \tau) d \tau,
$$

and

$$
G^{\prime \prime}(\omega)=\omega \int_{0}^{\infty} G(\tau) \cos (\omega \tau) d \tau,
$$

respectively, where $G(\tau)$ is the relaxation modulus in the following Boltzmann model of linear viscoelasticity

$$
\begin{aligned}
\sigma(t) & =\int_{-\infty}^{t} G(t-\tau) \dot{\gamma}(\tau) d \tau \\
& =\int_{0}^{\infty} G(\tau) \dot{\gamma}(t-\tau) d \tau
\end{aligned}
$$

with the stress $\sigma(t)$ satisfying

$$
\sigma(t)=G^{\prime}(\omega) \sin (\omega t)+G^{\prime \prime}(\omega) \cos (\omega t),
$$

when the strain $\gamma(t)$ corresponds to oscillatory shearing $\sin (\omega t)$, where $\omega$ denotes the frequency of the shearing. For the loss modulus $G^{\prime \prime}(\omega)$ (equation (17)), the choice of the Kohlrausch function as the relaxation modulus yields

$$
\begin{aligned}
G^{\prime \prime}(\omega) & =\omega \int_{0}^{\infty} \exp \left(-\tau^{\beta}\right) \cos (\omega \tau) d \tau \\
& =\pi \omega Q_{\beta}(\omega)
\end{aligned}
$$


where

$$
\begin{aligned}
Q_{\beta}(\omega) & =\frac{1}{2 \pi} \int_{-\infty}^{\infty} \exp \left(-i \omega \tau-|\tau|^{\beta}\right) d \tau \\
& =\frac{1}{\pi} \int_{0}^{\infty} \exp \left(-\tau^{\beta}\right) \cos (\omega \tau) d \tau
\end{aligned}
$$

is the symmetric (Lévy) stable distribution $[4,16]$. For stable distributions, $\beta$ lies in the range $0<\beta \leq 2$, which is larger than that required for the definition of the Kohlrausch function. Because of the known unimodal behaviour of the (Lévy) stable distributions [25], this analytic representation is consistent with situations where the loss modulus has a unimodal structure. Two Kohlrausch functions could be used when $G^{\prime \prime}(\omega)$ has two peaks. Consequently, to guarantee a strictly unimodal $G^{\prime \prime}(\omega)$, the choice of $\beta$ must be such that the linear growth of $\omega$ is slower than the decay of $Q_{\beta}(\omega)$ after it passes through the maximum. In fact, this is confirmed indicatively by the known asymptotic behaviour [16] of $Q_{\beta}(\omega)$

$$
\frac{\Gamma(1+\beta) \sin (\pi \beta / 2)}{\pi|\omega|^{\beta+1}} .
$$

The corresponding form for the storage modulus becomes

$$
G^{\prime}(\omega)=\omega \int_{0}^{\infty} \exp \left(-\tau^{\beta}\right) \sin (\omega \tau) d \tau .
$$

Some representative curves for different choices of $\beta$ are shown in Figure 1. They have a similar morphology to measured values of $G^{\prime}(\omega)$. This shows that the choice of the Kohlrausch function for the relaxation modulus is consistent with the known morphology of both $G^{\prime}(\omega)$ and $G^{\prime \prime}(\omega)$ for linear viscoelastic materials.

Alternatively, one can follow Bendler's analysis of dielectric relaxation [4] by assuming that $G(\tau)$ corresponds to the negative value of the derivative of a correlation function $\psi$ and that the correlation function is a Kohlrausch 

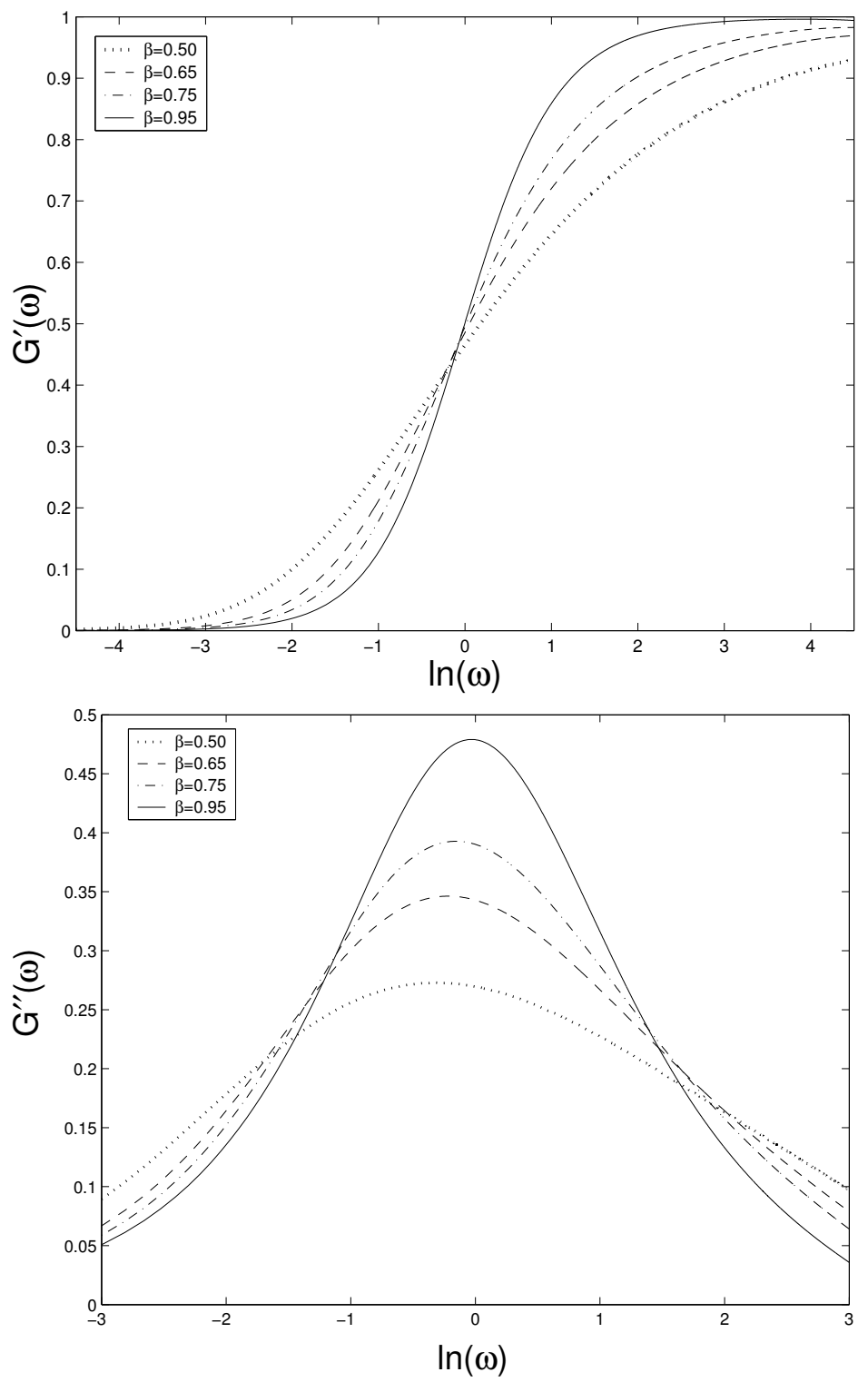

Figure 1: For $G(t)=\exp \left(-t^{\beta}\right)$, plots of $G^{\prime}(\omega)$ and $G^{\prime \prime}(\omega)$ with $\beta=0.50$, $0.65,0.75$ and 0.95 . 

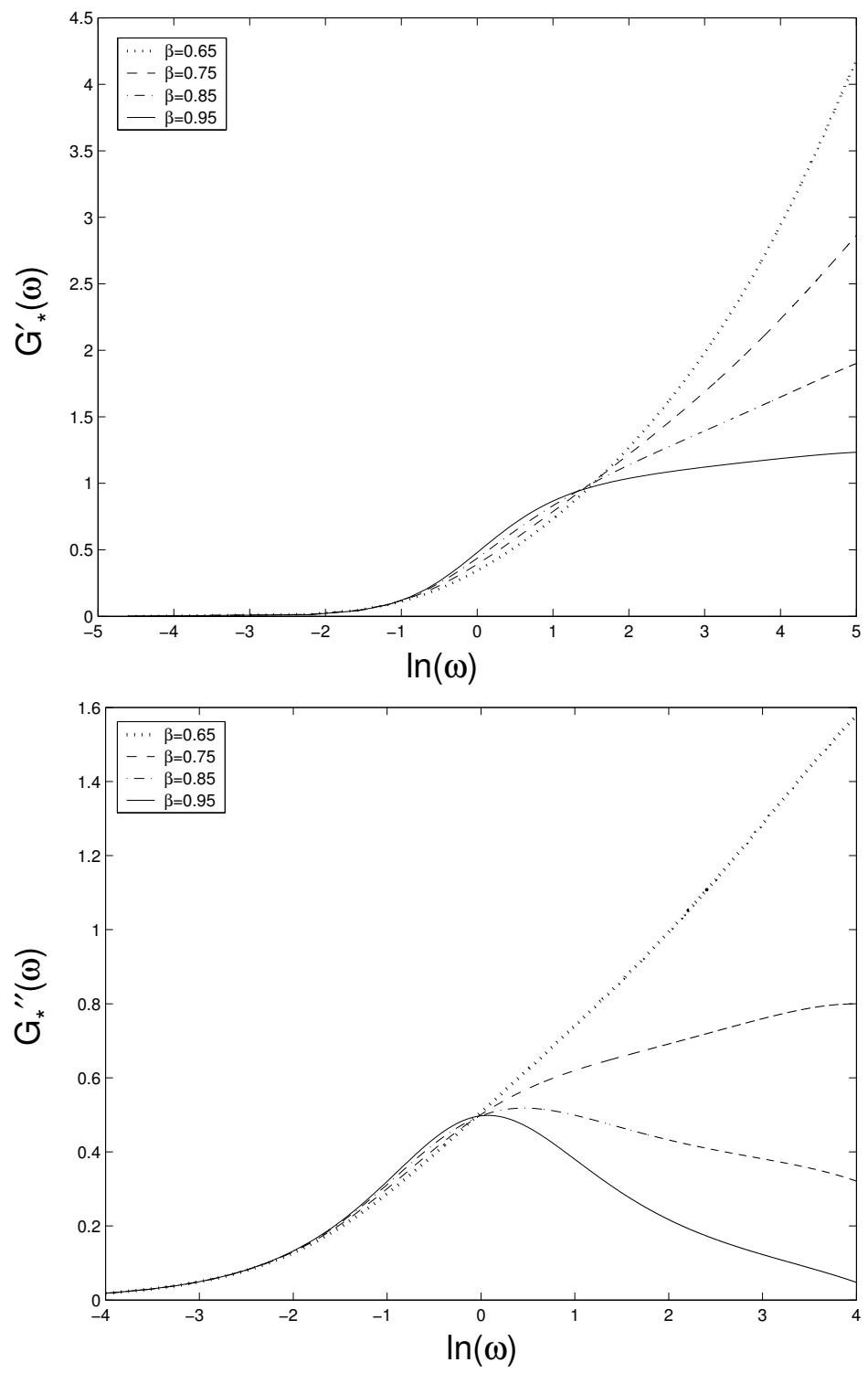

Figure 2: For $G(t)=\beta t^{\beta-1} \exp \left(-t^{\beta}\right)$, plots of $G_{*}^{\prime}(\omega)$ and $G_{*}^{\prime \prime}(\omega)$ with $\beta=$ $0.65,0.75,0.85$ and 0.95 . 
function. Substitution of this form for $G(t)$ in the oscillatory shear equations yields, for equation (26),

$$
\begin{aligned}
G_{*}^{\prime}(\omega) & =\pi \omega^{2} Q_{\beta}(\omega), \\
& =\beta \omega \int_{0}^{\infty} \tau^{\beta-1} \exp \left(-\tau^{\beta}\right) \sin (\omega \tau) d \tau .
\end{aligned}
$$

The corresponding form for $G^{\prime \prime}(\omega)$ of equation (21) becomes

$$
\begin{aligned}
G_{*}^{\prime \prime}(\omega) & =\beta \omega \int_{0}^{\infty} \tau^{\beta-1} \exp \left(-\tau^{\beta}\right) \cos (\omega \tau) d \tau, \\
& =\omega-\omega^{2} \int_{0}^{\infty} \exp \left(-\tau^{\beta}\right) \sin (\omega \tau) d \tau .
\end{aligned}
$$

Now, however, the choice of $\beta$ becomes more challenging. One the one hand, $\beta$ must be such that the linear growth of $\omega$ is faster than the decay of $Q_{\beta}(\omega)$ after it passes through its maximum in order to guarantee that the corresponding form of $G_{*}^{\prime}(\omega)$ has a morphology similar to that observed experimentally. On the other hand, the choice of $\beta$ must be such that the corresponding $G_{*}^{\prime \prime}(\omega)$ has the correct loss modulus morphology. It is clear that, for the representatives curves for different choices of $\beta$ shown in Figure 2, the Bendler choice for $G(t)$ limits the range of $\beta$ that guarantees that $G_{*}^{\prime \prime}(\omega)$ is strictly unimodal. This requires further investigation. In addition, the corresponding $G_{*}^{\prime}(\omega)$ can have a very strong growth because of the $\omega^{2}$ term in equation (27), given the asymptotic result (25).

\subsection{Large-scale polymer chain dynamics}

In a recent paper, De Gennes [5] observed that, as well as explaining the local segmental motion of a polymer near its glass transition, the Kohlrausch function can also model the large-scale chain dynamics far above the glass transition temperature as found in polydisperse systems. In addition, he hypothesizes that the exponent $\beta$ is sensitive to the presence or absence 
of entanglements. De Gennes establishes the relevance of the Kohlrausch function in the following manner. Let the memory function $M(t)$ be the Laplace transform of a distribution of relaxation rates $f$,

$$
M(t)=\int_{0}^{\infty} W(f) \exp (-f t) d f .
$$

On the basis of experimental and theoretical evidence for relaxation rates for polydisperse polymers as well as other situations, it can be confidently assumed that the behaviour of $W(f)$ often has the form

$$
W(f) \sim \exp \left(-1 / f^{\alpha}\right), \quad \alpha>0,
$$

which falls sharply for small $f$. For example, in the reptation dynamics for entangled chains, the distribution of $W(f)$ is given by

$$
W(f) \sim \exp \left(-\frac{1}{\bar{N}}\left(N_{e} / f \tau_{0}\right)^{1 / 3}\right),
$$

where $\bar{N}$ is the average molecular weight, $N_{e}$ is the distance between entanglements (with $N$, the polymerisation index, satisfying $N>N_{e}$ ), $f$ is the relaxation rate and $\tau_{0}$ is the microscopic jump time (independent of $N$ ). For $W(f)$ of equation (32), equation (31) takes the form

$$
M(t) \sim \int_{0}^{\infty} \exp (-S(f, t)) d f,
$$

where $S(f, t)=1 / f^{\alpha}+f t$. Differentiating $S(f, t)$ with respect to $f$, one obtains that at the minimum

$$
S \sim C t^{\beta}, \quad \beta=\frac{\alpha}{\alpha+1},
$$

where $C$ is a constant. On rewriting (34) as

$$
M(t) \sim \exp \left(-C t^{\beta}\right) \int_{0}^{\infty} \exp \left[-S(f, t)+C t^{\beta}\right] d f,
$$

and observing that the integral is bounded for suitably large $t$, it follows that

$$
M(t) \sim \exp \left(-C t^{\beta}\right) .
$$




\subsection{Recovery of Kohlrausch parameters}

Despite the wide applicability of Kohlrausch functions, little has been published about their use as a model for the relaxation modulus in the Boltzmann model (18). An exception is Schröter and Donth [23], who examined the merits of such a choice in some detail. For a single Kohlrausch function $K_{\tau, \beta}(t)$, such a choice reduces the problem of determining the relaxation modulus from measured values of the stress $\sigma(t)$ and the strain $\gamma(t)$ to determining the parameters of $\tau$ and $\beta$. Husain and Anderssen [10] established how this can be achieved. With the strain first applied at time $t=0$, they showed that the (standard) monomial moments of the Boltzmann equation (18), given by

$$
M_{p}=\int_{0}^{\infty} t^{p} \sigma_{L}(t) d t=\int_{0}^{\infty} t^{p}\left[\int_{0}^{t} \exp \left(-\left((t-\tau) / \tau_{0}\right)^{\beta}\right) \dot{\gamma}(\tau) d \tau\right] d t
$$

can be reorganised, using a change in the order of integration, to yield

$$
M_{p}=\frac{\tau_{0}}{\beta} \sum_{i=0}^{p}\left(\begin{array}{c}
p \\
i
\end{array}\right) \tau_{0}^{i} \Gamma\left(\frac{i+1}{\beta}\right) \int_{0}^{\infty} \tau^{p-i} \dot{\gamma}(\tau) d \tau,
$$

where $\Gamma(z), \Re(z)>0$, denotes the standard gamma function. This relationship leads naturally to simple algorithms for the recovery of estimates of $\tau_{0}$ and $\beta$ from moments defined on the measured values of $\sigma(t)$ and $\gamma(t)$. Related expressions for averaged moments of the relaxation time $\tau_{0}$ can be found in Lindsey and Patterson $[13, \S I V]$.

\subsection{Public domain algorithms}

As already mentioned, the Kohlrausch model for the relaxation modulus is quite popular in the study of a variety of physical and chemical processes. The new version of the Complex Nonlinear Least Squares (CNLS) 
frequency-response fitting software, LEVMw, v8.0, is now available (May 2003) at no cost, from Solartron instruments at the website http://www. solartronanalytical.com/downloads/software.html. It includes both the LEVM program files for MS-DOS and the new LEVMW files for full operation in WINDOWs. The material is provided in compressed, self-extracting form. Some of the programs in LEVM/LEVMW use the modified LevenbergMarquardt algorithm of Moré, which is robust, fast converging and involves implicitly scaled variables. The computer package allows accurate calculation of both temporal and frequency Kohlrausch response for arbitrary $\beta$. More information about the field may be found at http://www.physics. unc. edu $/{ }^{\sim} \operatorname{macd}$.

Acknowledgments: The authors acknowledge the wise and constructive advice of J. Ross Macdonald as well as an anonymous referee.

\section{References}

[1] R. S. Anderssen and R. J. Loy. Completely Monotone Fading Memory Relaxation Modulii. Bull. Austral. Math. Soc., 65:449-460, 2002. C803

[2] R. S. Anderssen and R. J. Loy. Rheological Implications of Completely Monotone Fading Memory. Journal of Rheology, 46(6):1459-1472, 2002. C803

[3] M. Avellaneda, R. Ryan and E. Weinan. PDFs for Velocity and Velocity Gradients in Burger's Turbulence. Phys. Fluids, 7(12):3067-3071, 1995. C802

[4] J. T. Bendler. Lévy (Stable) Probability Densities and Mechanical Relaxation in Solid Polymers. Journal of Statistical Physics, 36(5/6):625-637, 1984. C802, C805, C807, C809 
[5] P-G De Gennes. Relaxation Anomalies in Linear Polymer Melts. Macromolecules, 35:3785-3786, 2002. C801, C811

[6] G. Doetsch. Theorie und Anwendung der Laplace-Transformationen. Dover Publication, N. Y., 1943. C804

[7] W. Feller. An Introduction to Probability Theory and its Applications. John Wiley and Sons, New York, 2, 1970. C803

[8] A. Z. Grinshpan, M. E. H. Ismail, and D. L. Milligan. On Complete Monotonocity and Diesel Fuel Spray. Math. Intelligencer, 22:43-53, 2000. C803

[9] D. R. Holt and E. L. Crow. Tables and Graphs of the Stable Probability Density Functions. J. Res. Nat. Bur. Stand. Ser. B, 77B:143-198, 1973. C805

[10] Saiful A. Husain and R. S. Anderssen. Modelling the Relaxation Modulus of Linear Viscoelasticity Using the Kohlrausch Function. 2003 (In Preparation) C812

[11] P. Humbert. Nouvelles correspondances symboliques. Bull. Soc. Math. France, 69:121-129, 1945. C804

[12] R. Kohlrausch. Theorie des Elektrischen Rückstandes in der Leidener Flasche. Pogg. Ann. Phys. Chem., 91:179-214, 1854. C802

[13] C. P. Lindsey and G. D. Patterson. Detailed Comparison of the William-Watts and Cole-Davidson Functions. J. Chem. Phys., 73(7):3348-3357, 1980. C805, C812

[14] J. R. Macdonald. Paley-Wiener criterion for relaxation functions. J. Appl. Phys., 82:1476-1478, 1997. C806

[15] J. R. Macdonald. On Relaxation-Spectrum Estimation for Decades of Data: Accuracy and Sampling-Localization Considerations. Inverse Problems, 16:1561-1583, 2000. C802 
[16] E. W. Montroll and J. T. Bendler. On Lévy (or Stable) Distributions and the Williams-Watts Model of Dielectric Relaxation. Journal of Statistical Physics, 34:129-162, 1984. C801, C802, C804, C807

[17] K. L. Ngai, A. K. Rajagopal, R. W. Rendell and S. Teitler. Paley-Wiener Criterion for Relaxation Functions. Phys. Rev. B, 28(10):6073-6075, 1983. C805

[18] K. L. Ngai and C. M. Roland. Development of cooperativity in the local segmental dynamics of poly(vinylacetate): Synergy of thermodynamics and intermolecular coupling. Polymer, 43:567-573, 2002. C801

[19] R. Paley and N. Wiener. Fourier Transforms in the Complex Domain. Am. Math. Soc., Providence, R.I., Thm XII: p16, 1934. C805

[20] H. Pollard. The Representation of $e^{-x^{\lambda}}$ as a Laplace integral. Bull. Am. Math. Soc., 52:908-910, 1946. C803, C804

[21] R. Rambousky, M. Weiss, H. Mysz, M. Moske, and K. Samwer. Structural Relaxation and Viscous Flow in Amorphous $\mathrm{ZrAlCu}$ above and below the glass transition temperature. Mater. Sci. Forum, 225-227:83-88, 1996. C801

[22] N. Sasaki, Y. Nakayama, M. Yoshikawa and A. Enyo. Stress Relaxation Function of Bone and Bone Collagen. Journal of Biomechanics, 26(12):1369-1376, 1993. C801

[23] K. Schröter and E. Donth. Comparison of Shear Response with Other Properties at the Dynamic Glass Transition of Different Glassformers. Journal of Non-Crystalline Solids, 307-310:270-280, 2002. C812

[24] M. F. Shlesinger and E. W. Montroll. On the William-Watts function of Dielectric Relaxation. Proc. Natl. Acad. Sci. USA, 81:1280-1283, 1984. C801 
[25] V. M. Zolotarev. One-dimensional Stable Distributions. Translation of Mathematical Monographs, Am. Math. Soc., R.I., 65, 1986. C803, C807 